'Laboratorio de Oncología y Genética Molecular, Clínica Las Condes. Santiago, Chile. 2Unidad de Coloproctología, Clínica Las Condes. Santiago, Chile. aioquímico. ${ }^{\mathrm{b}} \mathrm{PhD}$.

Trabajo no recibió financiamiento. Los autores declaran no tener conflictos de interés.

Recibido el 22 de julio de 2020 aceptado el 8 de octubre de 2020.

Correspondencia a: Ana María Wielandt Lo Fontecilla 441, Las Condes. Santiago, Chile. awielandt@clc.cl

\section{Test de sangre oculta en deposiciones para programas de cribado de cáncer colorrectal: actualización}

\author{
ANA MARÍA WIELANDT ${ }^{1,2, a}$, CLAUDIA HURTADO ${ }^{1,2, a, b}$, \\ MAURICIO MORENO ${ }^{1,2, a, b}$, ALEJANDRO ZÁRATE2, \\ FRANCISCO LÓPEZ-KÖSTNER ${ }^{2}$
}

\section{Fecal occult blood test for colorectal cancer screening}

Screening programs for colorectal cancer $(C R C)$ are standard in most developed countries because they reduce mortality and are cost-effective. Within them, colonoscopy allows to directly visualize the colon and remove neoplastic lesions. However, it is an expensive exam with low adherence in asymptomatic individuals. The fecal occult blood test (FOBT) is a low-cost and risk-free method for the user, which results in a high rate of adherence, explaining its use in most screening programs. This article analyzes the effectiveness of different fecal occult blood tests in screening programs. The main conclusions are that the sensitivity of the guaiac-based chemical test for the detection of colorectal cancer is lower than that observed with qualitative and quantitative immunological tests. Automated quantitative methods allow objective readings independent of the operator and the reaction reading time, necessary for the analysis of large numbers of samples. The participation rate with immunological FOBTs is higher than with chemical ones, which is why they are preferred by the different countries that have screening programs. The use of quantitative tests allows stratification of symptomatic and asymptomatic patients at higher risk, in the screening programs.

(Rev Med Chile 2021; 149: 580-590)

Key words: Colorectal Neoplasms; Clinical Laboratory Techniques; Early Detection of Cancer; Immunological Test; Occult Blood.

\section{L} os programas de cribado hoy en día son un estándar en la mayoría de los países desarrollados porque reducen la mortalidad por cáncer colorrectal (CCR) y está demostrada su costo efectividad. Tanto en el mundo como en Chile, el CCR ha ido en aumento, por lo que el desarrollo de buenos programas de cribado se hace fundamental ${ }^{1-8}$. Por ello, la determinación de la cantidad de hemoglobina en las deposiciones es un método eficaz para el cribado del CCR en población asintomática y se ha preferido en programas de cribado tanto en países asiáticos como occidentales frente a otros métodos más invasivos o costosos $^{9-15}$.
Existe una amplia diversidad de estudios que evalúan la eficacia de los distintos test de sangre oculta en deposiciones (TSOD); sin embargo, muchos de ellos utilizan distintos parámetros lo cual dificulta su comparación. El objetivo de este trabajo es describir las características y analizar los distintos TSOD disponibles en el mercado nacional para evaluar su efectividad en un programa de cribado.

\section{Proceso de selección}

Se realizó una búsqueda en la literatura incluida en Cochrane Library, PUBMED, Lilacs, SciELO, Trip y Epistemonikos, utilizando como 
palabras claves: Screening, Tamizaje o Cribado de CCR; FOBT (fecal occult blood test) o TSOD (test de sangre oculta en deposiciones); FIT (Fecal immunological Test) o test inmunológico de sangre oculta en deposiciones; Test de Guayaco; para artículos en español o inglés. Se consideraron 175 artículos de los cuales se seleccionaron 74 para la revisión. Se incluyeron aquellas referencias más relevantes que permitieran realizar la comparación entre los distintos trabajos según características de indicadores de precisión de los test. Además, se buscó en el sitio web de Chile: www.chilecompra. cl los distintos test ofrecidos en el mercado chileno por licitaciones de distintas empresas y se revisó la información proporcionada por los laboratorios en sus manuales con respecto a las características de cada test ofrecido.

\section{Hemorragias ocultas}

La hemorragia oculta intestinal se define como la existencia de sangre no observable a simple vista en las heces, por lo tanto, indetectable para el paciente o el médico. Esta hemorragia se detecta con los test de sangre oculta en deposiciones $(\mathrm{TSOD})^{16}$. El principio de las técnicas en general es detectar hemoglobina ( $\mathrm{Hb}$ ). La Hb es una proteína formada por cuatro cadenas polipeptídicas ( $\alpha$ y $\beta$ globinas) a cada una de las cuales se une un grupo Hem.

$\mathrm{La} \mathrm{Hb}$ se fragmenta en el intestino produciendo diversos metabolitos que pueden o no ser detectados por los distintos TSOD. Las globinas son degradadas en el intestino delgado por proteasas disminuyendo progresivamente su concentración en el lumen intestinal y al llegar al colon derecho son metabolizadas por bacterias intestinales. Por su parte, el grupo Hem se metaboliza recién al llegar al colon derecho por las bacterias generando derivados porfirínicos. Esto explica que tanto las globinas, como los grupos Hem detectados por los TSOD provendrían principalmente del colon izquierdo o del recto ${ }^{17}$.

Dentro de los métodos para detectar hemoglobina están los químicos y los inmunológicos, y éstos a su vez pueden ser cromatográficos de captura tipo sándwich o inmunoensayos de aglutinación.

\section{Test químicos}

El test de sangre oculta en deposiciones basado en guayaco (TSODg) fue introducido hace más de 30 años en el mercado y aprobado en EE.UU. por la Food and Drug Administration (FDA); en la actualidad se comercializa principalmente como Hemoccult y Hemoccult-SENSA (Beckman Coulter, Fullerton, CA) siendo el último uno de los más usados dentro de los test químicos. Es una prueba cualitativa, sencilla de realizar y de bajo costo. Detecta $\mathrm{Hb}$ y grupo $\mathrm{Hem}$, por lo que tiene una baja sensibilidad en la hemorragia oculta de tracto gastrointestinal superior y derecho ${ }^{17}$.

$\mathrm{Su}$ fundamento es el cambio de coloración del sustrato que se produce por una reacción de oxidación de un compuesto fenólico como el ácido $\alpha$-guayacónico (guayaco) al agregar peróxido de hidrógeno, debido a la actividad peroxidasa o pseudoperoxidasa del grupo Hem de la hemoglobina. Por esta razón algunos alimentos que poseen actividad peroxidasa como frutas, vegetales, carnes rojas, y ciertos medicamentos irritantes de la mucosa gastrointestinal como antiinflamatorios no esteroideos (AINES) tales como ibuprofeno, indometacina, naproxeno, fenilbutazona y ácido acetilsalicílico; corticosteroides; reserpina o anticoagulantes, pueden provocar falsos positivos ${ }^{18}$.

Por el contrario, el consumo de vitamina $\mathrm{C}$ (ácido ascórbico) o determinados fármacos como cimetidina, sucralfato y omeprazol, utilizados para el tratamiento de úlceras o reflujo pueden conducir a resultados falsamente negativos (Beckman Coulter). Se puede aumentar la sensibilidad del test hidratando el frotis fecal, pero no se recomienda porque disminuye significativamente la especificidad causado por la activación de peroxidasas inespecíficas ${ }^{17,19,20}$. El colegio americano de gastroenterología (ACG) y la organización mundial de endoscopía (WEO) han recomendado dejar de utilizar los test de guayaco y optar por test inmunológicos en programas de cribado debido a su baja eficiencia ${ }^{21-24}$.

\section{Test inmunológicos}

El TSOD inmunológico (TSODi) permite medir la presencia de sangre mediante la reacción de un anticuerpo específico contra $\mathrm{Hb}$ humana. Tiene ventajas frente a los métodos químicos siendo simple, no invasivo, económico, no requiere restringir fármacos ni realizar dieta previa a la toma de la muestra, demostrando tener alta sensibilidad y especificidad para detectar $\mathrm{CCR}^{25}$. 
Existe una gran variedad de empresas que comercializan diversos test inmunológicos cualitativos y cuantitativos que han sido aprobados por la $\mathrm{FDA}^{26}$. Entre los métodos inmunológicos cualitativos en el mercado nacional se encuentran los cromatográficos como HemoSelect, Hemoccult ICT, SD-Bioline-FOB, ABON-FOB, BioNexia-FOB; Artron-FOB, Hexagon-OBTI y entre los cuantitativos los más utilizados mundialmente son los aglutinación por látex como el OC-Sensor (Eiken) y FOB-Gold (Sentinel) y aglutinación magnética como el Magstream (Fujireibo). OC-Sensor (Eiken) fue el único test cuantitativo disponible en el mercado nacional a partir del año 2007 (Tabla 1).
La elección del mejor test para el cribado de la población general es complejo puesto que existen muchos disponibles en el mercado y es difícil de comparar el rendimiento entre ellos. Lo anterior es debido a que el punto de corte para los test inmunológicos está expresado en concentración, es decir, cantidad de $\mathrm{Hb}$ en nanogramos (ng) por mililitro ( $\mathrm{ml}$ ) del reactivo contenido en el tubo de recolección (ng $\mathrm{Hb} / \mathrm{ml}$ ); y en el caso de los test químicos en microgramos ( $\mu \mathrm{g}) \mu \mathrm{g} \mathrm{Hb} / \mathrm{g}$ de deposición, por lo cual la concentración final depende del contenido de $\mathrm{Hb}$ por gramo de deposición, del volumen de reactivo del tubo de recolección y de la cantidad de deposición introducida al tubo que es variable para cada test.

Tabla 1. Principales Test de Sangre Oculta en Deposiciones presentes en el mercado nacional

\begin{tabular}{|c|c|c|c|c|c|c|}
\hline TSOD & Método & $\begin{array}{l}\text { Tipo de } \\
\text { lectura }\end{array}$ & $\begin{array}{l}\text { Límite } \\
\text { de corte }\end{array}$ & $\begin{array}{c}\text { n de } \\
\text { muestras }\end{array}$ & $\begin{array}{l}\text { Positividad } \\
\text { (\%) }\end{array}$ & Referencia \\
\hline \multicolumn{7}{|l|}{ Químicos } \\
\hline Hemoccult II (Beckman Coulter) & $\begin{array}{l}\text { Químico/pe- } \\
\text { roxidasa }\end{array}$ & Visual & $600 \mu \mathrm{g} / \mathrm{g}$ & 3 & $2,4-7,9$ & $(26-36)$ \\
\hline $\begin{array}{l}\text { Hemoccult Sensa (Beckman } \\
\text { Coulter) }\end{array}$ & $\begin{array}{l}\text { Químico/pe- } \\
\text { roxidasa }\end{array}$ & Visual & $300 \mu \mathrm{g} / \mathrm{g}$ & 3 & $4-13,6$ & $(29,37,38)$ \\
\hline \multicolumn{7}{|l|}{ Inmunológicos cualitativos } \\
\hline OC-Light (Eiken) & $\begin{array}{l}\text { Inmunocro- } \\
\text { matográfico }\end{array}$ & Visual & $50 \mathrm{ng} / \mathrm{ml}$ & 1 & $8,4-14,2$ & $(39,40)$ \\
\hline Abon-FOB/Spinreact (Tecnigen) & $\begin{array}{l}\text { Inmunocro- } \\
\text { matográfico }\end{array}$ & Visual & $50 \mathrm{ng} / \mathrm{ml}$ & 1 & NR & \\
\hline SD Bioline FOB (Medicatec) & $\begin{array}{l}\text { Inmunocro- } \\
\text { matográfico }\end{array}$ & Visual & $50 \mathrm{ng} / \mathrm{ml}$ & 1 & NR & \\
\hline BioNexia-FOB (Biomerieux) & $\begin{array}{l}\text { Inmunocro- } \\
\text { matográfico }\end{array}$ & Visual & $40 \mathrm{ng} / \mathrm{ml}$ & 1 & 23,5 & $(41)$ \\
\hline FOB-Test Artron-Canadá & $\begin{array}{l}\text { Inmunocro- } \\
\text { matográfico }\end{array}$ & Visual & $50 \mathrm{ng} / \mathrm{ml}$ & 1 a 3 & NR & \\
\hline Hexagon OBTI (Farmalatina) & $\begin{array}{l}\text { Inmunocro- } \\
\text { matográfico }\end{array}$ & Visual & $50 \mathrm{ng} / \mathrm{ml}$ & 1 & $35,8^{*}$ & $(42)$ \\
\hline \multicolumn{7}{|l|}{ Inmunológicos cuantitativos } \\
\hline FOB-Gold (Sentinel) & $\begin{array}{l}\text { Aglutinación } \\
\text { por látex }\end{array}$ & Automatizado & $117 \mathrm{ng} / \mathrm{ml}$ & 1 & $5,2-17,5$ & $(25,43-45)$ \\
\hline OC-Sensor; OC-Micro (Eiken) & $\begin{array}{l}\text { Aglutinación } \\
\text { por látex }\end{array}$ & Automatizado & $100 \mathrm{ng} / \mathrm{ml}$ & 2 & $4,8-11,2$ & $(27,36)$ \\
\hline $\begin{array}{l}\text { Immudia RPH (Magstream 1000) } \\
\text { (Fujireibo) }\end{array}$ & $\begin{array}{l}\text { Aglutinación } \\
\text { magnética }\end{array}$ & Automatizado & $20 \mathrm{ng} / \mathrm{ml}$ & 1 & $4,6-8,7$ & $(25,47-49)$ \\
\hline OC-Sensor; OC-Micro (Eiken) & $\begin{array}{l}\text { Aglutinación } \\
\text { por látex }\end{array}$ & Automatizado & $50 \mathrm{ng} / \mathrm{ml}$ & 2 & 19 & $\begin{array}{c}\text { Observación } \\
\text { propia }\end{array}$ \\
\hline OC-Sensor; OC-Micro (Eiken) & $\begin{array}{l}\text { Aglutinación } \\
\text { por látex }\end{array}$ & Automatizado & $100 \mathrm{ng} / \mathrm{ml}$ & 2 & 13 & $\begin{array}{l}\text { Observación } \\
\text { propia }\end{array}$ \\
\hline
\end{tabular}

TSOD: Test de Sangre Oculta e Deposiciones; NR: no reportado; *estudio realizado en población sintomática. 
Debido a que estas diferencias han causado confusión, se propuso estandarizar el reporte de $\mathrm{Hb}$ internacionalmente a $\mu \mathrm{g} \mathrm{Hb} / \mathrm{g}$ de deposición o a $\mu \mathrm{g} \mathrm{Hb} / \mathrm{ml}$ de deposición ${ }^{50,51}$ y así facilitar la comparación de los rendimientos; para ello los fabricantes deben reportar el volumen de deposición colectado en el tubo de muestra y el volumen de reactivo del tubo. Sin embargo, este último ( $\mu \mathrm{g} \mathrm{Hb} / \mathrm{ml}$ de deposición) aún no ha sido ampliamente implementado y dificulta aún más la comparación de distintas publicaciones realizadas con distintos test y distintos puntos de corte. Por ello, hasta el momento la mayoría de los trabajos han adoptado la medida $\mu \mathrm{g} \mathrm{Hb/g}$ de deposición. Por ejemplo, OC-Sensor considera el uso de 10 $\mathrm{mg}$ de deposición en 2,0 $\mathrm{ml}$ de reactivo, por lo tanto, un resultado de $100 \mathrm{ng} / \mathrm{ml}$ (punto de corte) es igual a $20 \mu \mathrm{g} / \mathrm{g}$ de deposición, en cambio FOBGold (Sentinel) utiliza 10 mg de deposición en 1,7 $\mathrm{ml}$ de reactivo por lo que $100 \mathrm{ng} / \mathrm{ml}$ es equivalente a $17 \mu \mathrm{g} / \mathrm{g}$ de deposición ${ }^{50}$ y Magstream reporta el uso de $0,3 \mathrm{mg}$ de deposición en $1 \mathrm{ml}$ de reactivo equivalentes a $67 \mu \mathrm{g} / \mathrm{g}$ de deposición (utilizado como punto de corte $)^{52}$.

Por otra parte, la positividad de las muestras puede estar afectada por variaciones de temperatura. La globina, componente de la $\mathrm{Hb}$, es susceptible a la degradación enzimática endógena o microbiana. Los TSODi dependen de la reacción entre los anticuerpos y la globina por lo cual utilizan un reactivo de estabilización en los tubos recolectores de las muestras para minimizar la degradación durante el tiempo entre la toma de la muestra y su análisis. Sin embargo, a pesar del uso de tampones estabilizadores, la concentración de hemoglobina decae en el tiempo llevando a falsos negativos. Se ha descrito que temperaturas elevadas pueden afectar la determinación, mostrando diferencias estacionales ${ }^{53}$. La congelación también afecta la estabilidad de la $\mathrm{Hb}$, donde se ha observado que múltiples ciclos de congelamiento y descongelamiento de las muestras afectan su estabilidad ${ }^{54}$.

Por lo tanto, la potencia diagnóstica del test para detectar lesiones estará dada por: número de muestras tomadas, la reproducibilidad y representatividad de la muestra, del punto de corte para la medida, estabilidad de la $\mathrm{Hb}$ en el tubo de recolección, variación de temperatura y tiempo de análisis desde la toma de la muestra ${ }^{55,56}$ y se mide con indicadores dados como Sensibilidad
(S), Especificidad (E), Valor Predictivo Positivo (VPP), Valor Predictivo Negativo (VPN), Eficacia (Ef) y la Razón de Verosimilitud (RV) ${ }^{26}$ (Tabla 2). Puesto que la prueba estándar de oro es la colonoscopía, estos parámetros estarán afectados por su calidad, ya que una colonoscopía de alta calidad permitirá aumentar la detección de pólipos de menor tamaño que se correlacionen con resultados positivos obtenidos con el TSOD. Se considera una colonoscopía de alta calidad la que cumple con altos estándares de los indicadores que son: obtener 7 o más puntos en la escala de Boston (preparación intestinal), llegada al ciego $y$ retiro mayor a $8 \mathrm{~min}$.

Se han reportado grandes diferencias de desempeño en cuanto a la potencia diagnóstica de distintos test inmunológicos cualitativos. La sensibilidad de los distintos test, aumenta con el tamaño de los adenomas, su número y su localización. Se ha descrito una sensibilidad entre un $11,4 \%$ y $58 \%$ para detectar adenomas en general y entre $25,4 \%$ a $71,5 \%$, para adenomas avanzados ${ }^{41}$. Adenoma avanzado fue definido como aquellos que presentan un tamaño $\geq 1 \mathrm{~cm}$ de diámetro, con componente velloso de $20 \%$ o más, o adenomas con alto grado de displasia. Al incluir pacientes con CCR al grupo de aquellos con adenomas avanzados Hundt et al. ${ }^{41}$, observan que la sensibilidad aumentó levemente y osciló entre 53,2\% y $73,8 \%$ con los distintos test cualitativos usados. Por otra parte, la especificidad fue de 58,8\% para adenomas y $96,7 \%$ para adenomas avanzados, con un VPP entre $38,4 \%$ y $60,5 \%$ y VPN entre $71,1 \%$ y $76 \%$ para cualquier adenoma según los distintos test utilizados. Además, se observó una fluctuación entre $5,1 \%$ a $50,6 \%$ de adenomas en localización proximal vs $12,6 \%$ a $59,8 \%$ con localización distal, respectivamente comparado al $6,1 \%$ y $4,2 \%$ detectado a nivel proximal y distal, respectivamente con test químico basado en guayaco ${ }^{41}$. En cuanto al número y tamaño de los adenomas, se observó una variación entre $7,8 \%$ y $53,3 \%$ de detección de 1 adenoma, $14 \%$ a $65 \%$ para la presencia de 2 adenomas y $24 \%$ a $68 \%$ para $\geq 3$ adenomas, con una variación según tamaño de $5,6 \%$ a $52 \%$ para adenomas $<1 \mathrm{~cm} \mathrm{y}$ $33,3 \%$ a $81 \%$ para adenomas $\geq 1 \mathrm{~cm}$; comparado con el 5,8\% detectado con test químico para 1 adenoma, $3,1 \%$ para 2 adenomas y $8,3 \%$ para $\geq 3$ adenomas con 3,3\% de ellos menores de $1 \mathrm{~cm}$ y $13,4 \%$ mayores de $1 \mathrm{~cm}^{41}$. 


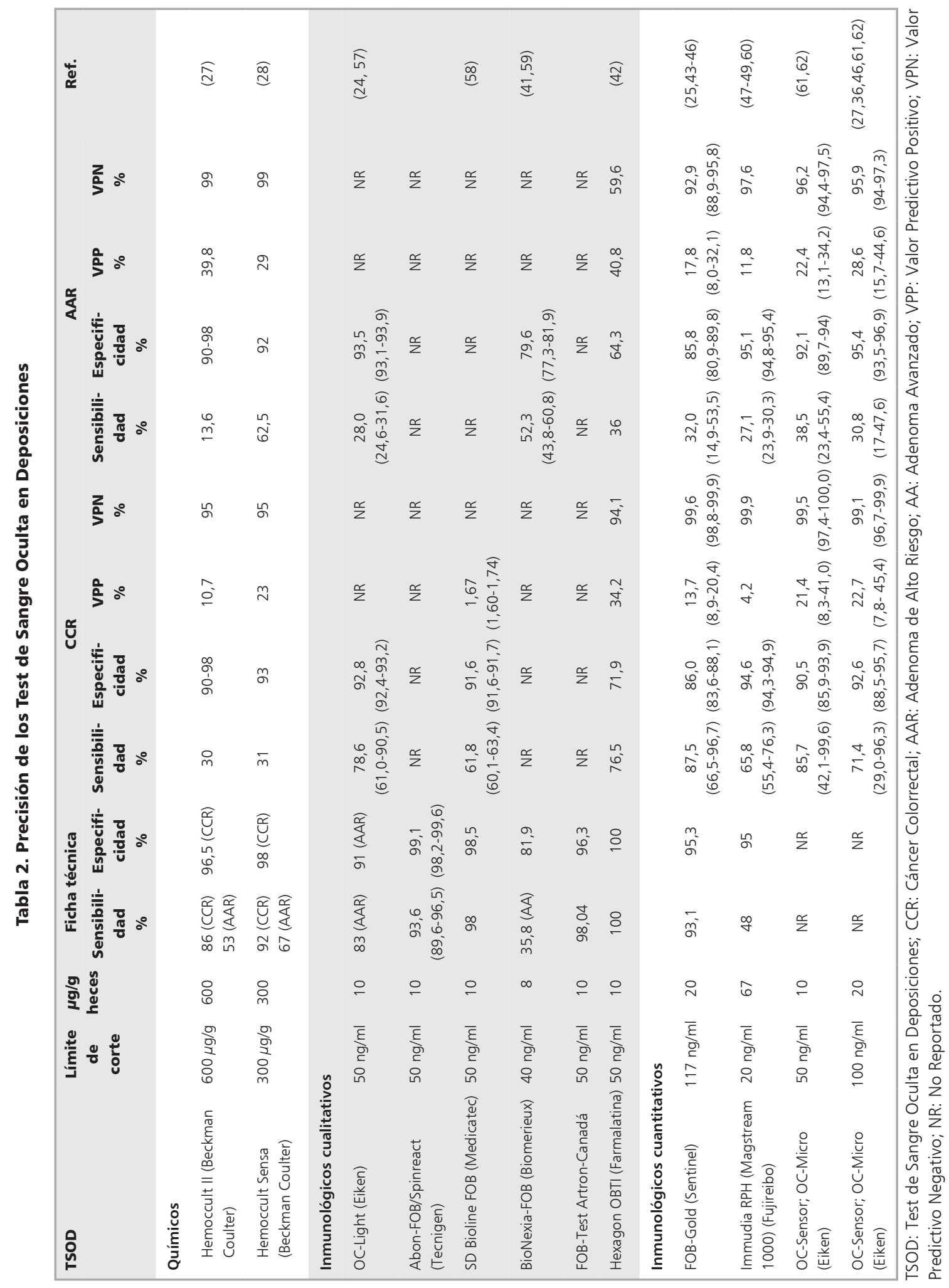


Por otra parte, varios estudios han demostrado que los métodos cuantitativos Magstream, FOB-Gold y OC-Sensor permiten aumentar la sensibilidad de detección de neoplasias avanzadas y adenomas comparadas con test de guayaco y los test inmunológicos cualitativos dado que la variación del punto de corte por ser cuantitativo permite un balance entre sensibilidad y especificidad (Tabla 2).

Comparando entre estos tres métodos cuantitativos se ha demostrado que OC-Sensor presenta una estabilidad similar a FOB-Gold en cuanto a la lectura de las muestras mantenidas a diferentes temperaturas ${ }^{27,45,63-66}$ (Tabla 3).

Las tasas de adherencia a la realización y seguimiento de los test han mejorado a través de los años, en la mayoría de los programas de cribado al implementar programas de capacitación y seguimiento a los pacientes. Lo anterior impacta directamente sobre la población, ya que el aumento en la incidencia y mortalidad por CCR, depende directamente de la realización periódica del test y por esa razón se han planteado distintas estrategias para incrementar las tasas de adherencia en estos programas $^{64}$ (Tabla 4).

La elección del TSOD a utilizar en los programas de cribado dependerá principalmente del rendimiento de cada test, sin embargo, se deben considerar otros factores como la aceptación de la población del test, su adherencia, el costo, el número de muestras a tomar y restricciones de dieta y fármacos tales como AINES, anticoagulantes, corticoides y vitamina C. Los test automatizados ofrecen además una serie de ventajas tales como la integración a sistemas informáticos de laboratorio, uso de códigos de barra para la identificación del paciente, gran capacidad de análisis para un alto volumen de muestras y control de calidad mediante curvas de calibración y diferentes controles de positividad (alta y baja concentración de $\mathrm{Hb}$ ), reinterpretación de resultados según patología o necesidad.

Tabla 3. Estabilidad de lectura a distintas temperaturas para OC-sensor vs FOB-Gold

\begin{tabular}{|c|c|c|c|c|c|}
\hline $\begin{array}{l}\text { Temperaturas } \\
\text { Tiempo }\end{array}$ & $-18^{\circ} \mathrm{C}$ a $-24^{\circ} \mathrm{C}$ & $4^{\circ} \mathrm{C}$ a $8^{\circ} \mathrm{C}$ & días $20^{\circ} \mathrm{C}$ a $26^{\circ} \mathrm{C}$ & $29^{\circ} \mathrm{C}$ a $35^{\circ} \mathrm{C}$ & Referencia \\
\hline OC-sensor & 10 a 14 & 7 & 3 & $<3$ & $(27)$ \\
\hline OC-sensor & NA & 7 & 4 & 5 & (64) \\
\hline FOB-Gold & NA & 4 & 4 & 5 & (64) \\
\hline FOB-Gold & $30-35$ & 32 & NA & 3 & $(66)$ \\
\hline OC-sensor & $30-35$ & 35 & 14 & NA & Observación propia \\
\hline
\end{tabular}

NA: No analizado.

Tabla 4. Comparación de cribado entre los TSOD y colonoscopía6,67

\begin{tabular}{|c|c|c|c|}
\hline Porcentaje & $\begin{array}{c}\text { TSODg } \\
\%\end{array}$ & $\begin{array}{c}\text { TSODi } \\
\%\end{array}$ & $\begin{array}{c}\text { Colonoscopía } \\
\%\end{array}$ \\
\hline Sensibilidad para AAR & $9-24$ & $32-53$ & $88-98$ \\
\hline Sensibilidad para CCR & $13-50$ & 79 & $92-99$ \\
\hline Tasa de participación & $16-47$ & $17-77$ & $16-93$ \\
\hline Tasa de positividad & $2,4-6,8$ & $1,1-13$ & $4,9-11$ \\
\hline Tasa de detección neoplasia avanzada & $29-50$ & $16-43$ & 100 \\
\hline Reducción incidencia de CCR (\%) & $17-20$ & 10 & 69 \\
\hline Reducción mortalidad de CCR (\%) & $9-22$ & $22-62$ & 68 \\
\hline
\end{tabular}

TSOD: Test de Sangre Oculta en Deposiciones; g: guayaco; i: inmunológico; AAR: Adenoma de Alto Riesgo; CCR: Cáncer colorrectal. 
Un punto de discusión ha sido también el uso de 1 o 2 muestras para realizar cribados poblacionales y si estos deben realizarse en forma anual o bienal ${ }^{68,69}$. Kaminski et al. ${ }^{69}$ reportan las consideraciones adoptadas por diversos programas de cribado en poblaciones a nivel mundial. De acuerdo con las instrucciones proporcionadas por los distintos fabricantes de los TSOD, se recomienda tomar 1 muestra en 1 día, excepto para el test químico basado en guayaco como el Hemoccult que requiere 3 días de muestras con restricción de dieta y medicamentos. Sin embargo, varios trabajos reportan una mejor sensibilidad de las pruebas inmunológicas al tomar 2 muestras y realizar los programas de cribado en forma anual, como van Roon et $\mathrm{al}^{70}$ que reportan una detección de neoplasia avanzada de 4,1\% [95\%CI, 3,3\% $5,1 \%$ ] vs $3,1 \%$ [ $95 \%$ CI, $2,5 \%-3,8 \%$ ] con 2 y 1 muestra respectivamente, concordando con lo reportado por Park et $\mathrm{al}^{36} \mathrm{y}$ Nakama ${ }^{71}$. No obstante, otros estudios no muestran diferencias, como Lee et $\mathrm{al}^{72}$ quienes describen una sensibilidad para la detección de CCR con 1, 2 o 3 muestras de 0,79 (95\% CI, 0,65 - 0,89) 0,77 (95\% CI, 0,59-0,89) y $0,80(95 \% \mathrm{CI}, 0,66$ - 0,89), respectivamente en población asintomática con una especificidad de 0,94 (95\% CI, 0,92 - 0,95), 0,93 (95\% CI, 0,90 0,95), 0,93 (95\% CI, 0,89 - 0,95), respectivamente concordando con Hernández ${ }^{73}$, Wong ${ }^{74}$, Guittet ${ }^{75}$, por lo cual las recomendaciones que favorezcan realizar el cribado con 1 muestra en forma anual después de un metaanálisis ${ }^{72}$ y una revisión sistemática sobre este punto ${ }^{13}$ en población asintomática son débiles y con baja evidencia por sobre el uso de 2 muestras ${ }^{72}$.

El número total de neoplasias avanzadas encontradas en la repetición del examen TSODi no está influenciado por la duración del intervalo dentro de un rango de 1-3 años. Además, hay una participación estable y aceptablemente alta en la segunda ronda de selección. Esto implica que los intervalos de detección pueden adaptarse a los recursos locales ${ }^{76}$. Además, no hay diferencia en la asistencia para los sujetos a los que se les ofrece una evaluación TSODi de 1 o 2 muestras ${ }^{72}$. Los resultados permiten el desarrollo de estrategias eficientes de detección TSODi que se pueden adaptar para las capacidades locales de colonoscopía, en lugar de variar el valor de corte en una estrategia de 1 muestra $^{70}$.

Considerando el aumento del CCR en Chile y las limitaciones económicas que existen para implementar un programa de cribado, la Unidad de Coloproctología de Clínica Las Condes, junto a Tokyo Medical and Dental University de Japón y al Ministerio de Salud de Chile desarrollaron un Programa de Prevención de Neoplasias Colorrectales (PRENEC) para población asintomática usuaria del sistema público de salud. El programa cuenta con un protocolo de seguimiento a los participantes además de instancias de educación con lo cual obtuvo una adherencia mayor a la reportada por experiencias internacionales ${ }^{10,15,77,78}$. Como resultado de los 5 primeros años de este programa de cribado con el test inmunológico OC-sensor se logró una adherencia de $92 \%$ al test con 26.444 pacientes asintomáticos enrolados. Se obtuvo un $15,1 \%$ de pacientes con test inmunológico positivo en los cuales se logró identificar $46,8 \%$ de pacientes con adenomas o adenocarcinomas y una tasa de detección de cáncer de $4,61 \%{ }^{78}$. La alta detección de adenomas de alto riesgo y cáncer apoya la conveniencia de realizar estos programas de cribado en el país.

\section{Conclusiones}

Existe una gran variación en el rendimiento de los test de los distintos fabricantes del mercado. Los métodos inmunológicos muestran mayor sensibilidad, especificidad, VPP y VPN que los químicos para la detección de lesiones colorrectales. De ellos, los cuantitativos por ser automatizados, permiten ser utilizados en programas de cribado con un gran número de participantes.

En relación a la adherencia de los pacientes a la realización del test inmunológico por sobre los químicos, esto se debe en parte a la necesidad de tomar 3 muestras consecutivas de deposiciones, dificultad en el manejo, almacenamiento de las muestras hasta su procesamiento y la necesidad de suspender fármacos o ciertos alimentos. Con respecto al número de muestras a solicitar, al punto de corte elegido y el intervalo de seguimiento de los pacientes, estos deben considerar los costos y la capacidad de resolución endoscópica que ello significa para el país o centro. Además, los métodos cuantitativos permiten la posibilidad de reinterpretar resultados por contar con una curva de calibración y definir el punto de corte según la población de estudio. La factibilidad de poder 
cambiar el punto de corte permite estratificar pacientes sintomáticos y asintomáticos de mayor riesgo viendo el costo y efectividad en los programas de cribado lo cual representa una ventaja frente a los métodos cualitativos.

\section{Referencias}

1. Bray F, Ferlay J, Soerjomataram I, Siegel RL, Torre LA, Jemal A. Global cancer statistics 2018: GLOBOCAN estimates of incidence and mortality worldwide for 36 cancers in 185 countries. CA Cancer J Clin. 2018; 68 (6): 394-424.

2. Arnold M, Sierra MS, Laversanne M, Soerjomataram I, Jemal A, Bray F. Global patterns and trends in colorectal cancer incidence and mortality. Gut. 2017; 66 (4): 68391.

3. Ministerio de Salud de Chile. Serie de defunciones, mortalidad cruda, mortalidad ajustada por tumores malignos seleccionados según sexo y región [Internet]. Disponible en: http://www.deis.cl/series-y-graficos-de-mortalidad/. Último acceso, mayo de 2020.

4. Zárate AJ, Alonso FT, Garmendia ML, López-Köstner F. Increasing crude and adjusted mortality rates for colorectal cancer in a developing South American country. Colorectal Dis. 2013; 15 (1): 47-51.

5. Frazier AL, Colditz GA, Fuchs CS, Kuntz KM. Cost-effectiveness of screening for colorectal cancer in the general population. JAMA 2000; 284: 1954-61.

6. Ladabaum U, Dominitz JA, Kahi C, Schoen RE. Strategies for Colorectal Cancer Screening. Gastroenterology. 2020; 158 (2): 418-32.

7. Mendivil J, Appierto M, Aceituno S, Comas M, Rué M. Economic evaluations of screening strategies for the early detection of colorectal cancer in the average-risk population: A systematic literature review. PLoS One. 2019; 14 (12): e0227251.

8. Gini A, Jansen EEL, Zielonke N, Meester RGS, Senore C, Anttila A, et al. EU-TOPIA consortium. Impact of colorectal cancer screening on cancer-specific mortality in Europe: A systematic review. Eur J Cancer. 2020; 127: 224-35. Review.

9. López-Köstner F, Kronberg U, Zárate AJ, Wielandt AM, Pinto E, Suazo C, et al. A screening program for colorectal cancer in Chilean subjects aged fifty years or more. Rev Med Chile 2012; 140 (3): 281-6.

10. Okada T, Tanaka K, Kawachi H, Ito T, Nishikage T, Odagaki $\mathrm{T}$, et al. International collaboration between Japan and Chile to improve detection rates in colorectal cancer screening. Cancer. 2016; 122 (1): 71-7.
11. Issa I, Noureddine M. Colorectal cancer screening: An updated review of the available options. World J Gastroenterol 2017; 23 (28): 5086-96.

12. Le Pimpec F, Moutel G, Piette C, Lièvre A, Bretagne JF. Fecal immunological blood test is more appealing than the guaiac-based test for colorectal cancer screening. Dig Liver Dis. 2017; 49 (11): 1267-72.

13. Robertson DJ, Lee JK, Boland CR, Dominitz JA, Giardiello FM, Johnson DA, et al. Recommendations on Fecal Immunochemical Testing to Screen for Colorectal Neoplasia: A Consensus Statement by the US Multi-Society Task Force on Colorectal Cancer. Gastroenterology. 2017; 152 (5): 1217-37.e3. Review.

14. Navarro M, Nicolas A, Ferrandez A, Lanas A. Colorectal cancer population screening programs worldwide in 2016: An update. World J Gastroenterol. 2017; 23 (20): 3632-42. Review.

15. López-Kostner F, Zárate AJ, Ponce A, Kronberg U, Kawachi H, Okada T, et al. Programa multicéntrico de cribado de cáncer colorrectal en Chile. [Results of a multicentric colorectal cancer screening program in Chile]. Rev Med Chile 2018; 146 (6): 685-92.

16. Zuckerman GR, Prakash C, Askin MP, Lewis BS. AGA technical review on the evaluation and management of occult and obscure gastrointestinal bleeding. Gastroenterology. 2000; 118 (1): 201-21.

17. Sempere L, Pérez-Mateo M. Práctica Clínica en Gastroenterología y Hepatología. Capítulo 5 Hemorragia Oculta Intestinal. Asociación Española de Gastroenterología, (CCTO EDITORIAL, S.L., 2016. Página Web: www.grupocto.es ISBN de la obra completa: 978-8415946-96-0 ISBN del Volumen I: 978-84-16403-97-4 Depósito legal: M-21093-2015 Impreso en España.

18. Pignone M, Campbell MK, Carr C, Phillips C. Meta-analysis of dietary restriction during fecal occult blood testing. Eff Clin Pract. 2001; 4 (4): 150-6.

19. Allison JE, Tekawa IS, Ransom LJ, Adrain AL. A comparison of fecal occult-blood tests for colorectal-cancer screening. N Engl J Med. 1996; 334 (3): 155-9.

20. Mandel JS, Bond JH, Church TR, Snover DC, Bradley GM, Schuman LM, et al. Reducing mortality from colorectal cancer by screening for fecal occult blood. Minnesota Colon Cancer Control Study. N Engl J Med. 1993; 328 (19): 1365-71.

21. Rex DK, Johnson DA, Anderson JC, Schoenfeld PS, Burke CA, Inadomi JM. American College of Gastroenterology. American College of Gastroenterology guidelines for colorectal cancer screening 2009 [published correction appears in Am J Gastroenterol. 2009; 104 (6): 1613]. Am J Gastroenterol. 2009; 104 (3): 739-50.

22. Allison JE, Fraser CG, Halloran SP, Young GP. Popu- 
lation screening for colorectal cancer means getting FIT: the past, present, and future of colorectal cancer screening using the fecal immunochemical test for hemoglobin (FIT). Gut Liver. 2014; 8 (2): 117-30.

23. Fraser CG, Allison JE, Young GP, Halloran SP, Seaman HE. Improving the reporting of evaluations of faecal immunochemical tests for haemoglobin: the FITTER standard and checklist. Eur J Cancer Prev. 2015; 24 (1): 24-6.

24. Lin JS, Piper MA, Perdue LA, Rutter C, Webber EM, O'Connor E, et al. Screening for Colorectal Cancer: A Systematic Review for the U.S. Preventive Services Task Force [Internet]. Rockville (MD): Agency for Healthcare Research and Quality (US) 2016. Report No.: 14-05203EF-1.

25. Faivre J, Dancourt V, Denis B, Dorval E, Piette C, Perrin $\mathrm{P}$, et al. Comparison between a guaiac and three immunochemical faecal occult blood tests in screening for colorectal cancer. Eur J Cancer. 2012; 48 (16): 2969-76.

26. Quintero E. ¿Test químico o test inmunológico para la detección de sangre oculta en heces en el cribado del cáncer colorrectal? [Chemical or immunological tests for the detection of fecal occult blood in colorectal cancer screening?]. Gastroenterol Hepatol. 2009; 32 (8): 565-76.

27. Rabeneck L, Rumble RB, Thompson F, Mills M, Oleschuk C, Whibley A, et al. Fecal immunochemical tests compared with guaiac fecal occult blood tests for population-based colorectal cancer screening. Can J Gastroenterol. 2012; 26 (3): 131-47. Review.

28. Rozen P, Knaani J, Samuel Z. Performance characteristics and comparison of two immunochemical and two guaiac fecal occult blood screening tests for colorectal neoplasia. Dig Dis Sci. 1997; 42 (10): 2064-71.

29. Allison JE, Tekawa IS, Ransom LJ, Adrain AL. A comparison of fecal occult-blood tests for colorectal-cancer screening. N Engl J Med. 1996; 334 (3): 155-9.

30. Castiglione G, Zappa M, Grazzini G, Mazzotta A, Biagini $\mathrm{M}$, Salvadori $\mathrm{P}$, et al. Immunochemical vs guaiac faecal occult blood tests in a population-based screening programme for colorectal cancer. Br J Cancer. 1996; 74 (1): $141-4$.

31. Young GP, St John DJ, Winawer SJ, Rozen P. WHO (World Health Organization) and OMED (World Organization for Digestive Endoscopy). Choice of fecal occult blood tests for colorectal cancer screening: recommendations based on performance characteristics in population studies: a WHO (World Health Organization) and OMED (World Organization for Digestive Endoscopy) report. Am J Gastroenterol. 2002; 97 (10): 2499-507.
32. Zappa M, Castiglione G, Paci E, Grazzini G, Rubeca $\mathrm{T}$, Turco P, et al. Measuring interval cancers in population-based screening using different assays of fecal occult blood testing: the District of Florence experience. Int J Cancer. 2001; 92 (1): 151-4.

33. Guittet L, Bouvier V, Mariotte N, Vallee JP, Arsène D, Boutreux S, et al. Comparison of a guaiac based and an immunochemical faecal occult blood test in screening for colorectal cancer in a general average risk population. Gut. 2007; 56 (2): 210-4.

34. van Rossum LG, van Rijn AF, Laheij RJ, van Oijen MG, Fockens $\mathrm{P}$, van Krieken $\mathrm{HH}$, et al. Random comparison of guaiac and immunochemical fecal occult blood tests for colorectal cancer in a screening population. Gastroenterology. 2008; 135 (1): 82-90.

35. Dancourt V, Lejeune C, Lepage C, Gailliard MC, Meny B, Faivre J. Immunochemical faecal occult blood tests are superior to guaiac-based tests for the detection of colorectal neoplasms. Eur J Cancer. 2008; 44 (15): 22548.

36. Park DI, Ryu S, Kim YH, Lee SH, Lee CK, Eun CS, et al. Comparison of guaiac-based and quantitative immunochemical fecal occult blood testing in a population at average risk undergoing colorectal cancer screening. Am J Gastroenterol. 2010; 105 (9): 2017-25.

37. Allison JE, Sakoda LC, Levin TR, Tucker JP, Tekawa IS, Cuff T, et al. Screening for colorectal neoplasms with new fecal occult blood tests: update on performance characteristics. J Natl Cancer Inst. 2007; 99 (19): 146270.

38. Smith A, Young GP, Cole SR, Bampton P. Comparison of a brush-sampling fecal immunochemical test for hemoglobin with a sensitive guaiac-based fecal occult blood test in detection of colorectal neoplasia. Cancer. 2006; 107 (9): 2152-9.

39. Alsayid M, Singh MH, Issaka R, Laleau V, Day L, Lee J, et al. Yield of Colonoscopy After a Positive Result From a Fecal Immunochemical Test OC-Light. Clin Gastroenterol Hepatol. 2018; 16 (10): 1593-7.

40. Pham R, Cross S, Fernández B, Corson K, Dillon K, Yackley C, et al. "Finding the Right FIT": Rural Patient Preferences for Fecal Immunochemical Test (FIT) Characteristics. J Am Board Fam Med. 2017; 30 (5): 632-44.

41. Hundt S, Haug U, Brenner H. Comparative evaluation of immunochemical fecal occult blood tests for colorectal adenoma detection. Ann Intern Med. 2009 Feb 3; 150 (3): 162-9.

42. Tusen Toledo Y, Chao González, L, Barroso Márquez, L. Valor de la prueba de sangre oculta en heces fecales para la detección de lesiones premalignas y malignas del 
colon. Revista Cubana de Medicina Militar. 2011; 40 (34): 234-40.

43. Graser A, Stieber P, Nagel D, Schäfer C, Horst D, Becker $\mathrm{CR}$, et al. Comparison of CT colonography, colonoscopy, sigmoidoscopy and faecal occult blood tests for the detection of advanced adenoma in an average risk population. Gut. 2009; 58 (2): 241-8.

44. Wieten E, de Klerk CM, van der Steen A, Ramakers CR, Kuipers EJ, Hansen BE, et al. Equivalent Accuracy of 2 Quantitative Fecal Immunochemical Tests in Detecting Advanced Neoplasia in an Organized Colorectal Cancer Screening Program. Gastroenterology. 2018; 155 (5): 1392-9.e5.

45. Dvir R, Clementi M. Sentifit 270 System clinical performance evaluation compared with OC-Sensor Diana, for the detection of Human Hemoglobin as Fecal Occult Blood (FOB). 2016, Poster P0345 United European Gastroenterology (UEG) week, Oct 15, Viena, Austria.

46. Auge JM, Rodríguez C, Espanyol O, Rivero L, Sandalinas S, Grau J, et al. An evaluation of the SENTiFIT 270 analyser for quantitation of faecal haemoglobin in the investigation of patients with suspected colorectal cancer. Clin Chem Lab Med. 2018; 56 (4): 625-33.

47. Morikawa T, Kato J, Yamaji Y, Wada R, Mitsushima $\mathrm{T}$, Shiratori Y. A comparison of the immunochemical fecal occult blood test and total colonoscopy in the asymptomatic population. Gastroenterology. 2005; 129 (2): $422-8$.

48. Wong CK, Fedorak RN, Prosser CI, Stewart ME, van Zanten SV, Sadowski DC. The sensitivity and specificity of guaiac and immunochemical fecal occult blood tests for the detection of advanced colonic adenomas and cancer. Int J Colorectal Dis. 2012; Dec; 27 (12): 1657-64.

49. Launoy GD, Bertrand HJ, Berchi C, Talbourdet VY, Guizard AV, Bouvier VM, et al. Evaluation of an immunochemical fecal occult blood test with automated reading in screening for colorectal cancer in a general average-risk population. Int J Cancer. 2005; 115 (3): 493-6. Erratum in: Int J Cancer. 2005; 116 (6): 1004.

50. Fraser CG, Allison JE, Halloran SP, Young GP; Expert Working Group on Fecal Immunochemical Tests for Hemoglobin, Colorectal Cancer Screening Committee, World Endoscopy Organization. A proposal to standardize reporting units for fecal immunochemical tests for hemoglobin. J Natl Cancer Inst. 2012; 104 (11): 810-4.

51. Fraser CG, Rapi S, Rubeca T. RE: A Proposal to Standardize Reporting Units for Fecal Immunochemical Tests for Hemoglobin. J Natl Cancer Inst. 2016; 108 (1): djv312.

52. Wong CK, Fedorak RN, Prosser CI, Stewart ME, van Zanten SV, Sadowski DC. The sensitivity and specificity of guaiac and immunochemical fecal occult blood tests for the detection of advanced colonic adenomas and cancer. Int J Colorectal Dis. 2012; 27 (12): 1657-64.

53. Grazzini G, Ventura L, Zappa M, Ciatto S, Confortini $\mathrm{M}$, Rapi S, et al. Influence of seasonal variations in ambient temperatures on performance of immunochemical faecal occult blood test for colorectal cancer screening: observational study from the Florence district. Gut. 2010; 59 (11): 1511-5.

54. Catomeris P, Baxter NN, Boss SC, Paszat LF, Rabeneck L, Randell E, et al. Effect of Temperature and Time on Fecal Hemoglobin Stability in 5 Fecal Immunochemical Test Methods and One Guaiac Method. Arch Pathol Lab Med. 2018; 142 (1): 75-82.

55. van Rossum LG, van Rijn AF, Laheij RJ, van Oijen MG, Fockens $\mathrm{P}$, Jansen JB, et al. Cutoff value determines the performance of a semi-quantitative immunochemical faecal occult blood test in a colorectal cancer screening programme. Br J Cancer. 2009; 101 (8): 1274-81.

56. Rozen P, Waked A, Vilkin A, Levi Z, Niv Y. Evaluation of a desk top instrument for the automated development and immunochemical quantification of fecal occult blood. Med Sci Monit. 2006; 12 (6): MT27-MT32.

57. Chiu HM, Lee YC, Tu CH, Chen CC, Tseng PH, Liang JT, et al. Association between early stage colon neoplas$\mathrm{ms}$ and false-negative results from the fecal immunochemical test. Clin Gastroenterol Hepatol. 2013; 11 (7): 832-8.e1-2.

58. Shin A, Choi KS, Jun JK, Noh DK, Suh M, Jung KW, et al. Validity of fecal occult blood test in the national cancer screening program, Korea. PLoS One. 2013; 8 (11): e79292.

59. Brenner H, Haug U, Hundt S. Inter-test agreement and quantitative cross-validation of immunochromatographical fecal occult blood tests. Int J Cancer. 2010; 127 (7): 1643-9.

60. Launois R, Le Moine JG, Uzzan B, Fiestas Navarrete LI, Benamouzig R. Systematic review and bivariate/HSROC random-effect meta-analysis of immunochemical and guaiac-based fecal occult blood tests for colorectal cancer screening. Eur J Gastroenterol Hepatol. 2014; 26 (9): 978-89.

61. Nicholson BD, James T, East JE, Grimshaw D, Paddon $\mathrm{M}$, Justice S, et al. Experience of adopting faecal immunochemical testing to meet the NICE colorectal cancer referral criteria for low-risk symptomatic primary care patients in Oxfordshire, UK. Frontline Gastroenterol. 2019; 10 (4): 347-55.

62. Ou CH, Kuo FC, Hsu WH, Lu CY, Yu FJ, Kuo CH, et al. Comparison of the performance of guaiac-based and two immunochemical fecal occult blood tests for 
identifying advanced colorectal neoplasia in Taiwan. J Dig Dis. 2013; 14 (9): 474-83.

63. Guittet L, Guillaume E, Levillain R, Beley P, Tichet J, Lantieri $\mathrm{O}$, et al. Analytical comparison of three quantitative immunochemical fecal occult blood tests for colorectal cancer screening. Cancer Epidemiol Biomarkers Prev. 2011; 20 (7): 1492-501.

64. Gies A, Cuk K, Schrotz-King P, Brenner H. Direct comparison of ten quantitative fecal immunochemical tests for hemoglobin stability in colorectal cancer screening. Clin Transl Gastroenterol. 2018; 9 (7):168.

65. Chen H, Werner S, Brenner H. Fresh vs Frozen Samples and Ambient Temperature Have Little Effect on Detection of Colorectal Cancer or Adenomas by a Fecal Immunochemical Test in a Colorectal Cancer Screening Cohort in Germany. Clin Gastroenterol Hepatol. 2017; 15 (10): 1547-56.e5.

66. Paparella C, Barazzutti A, Cugini A, Lucini R. Stability of human hemoglobin in fecal samples at different storage conditions in fecal sampling device for colorectal cancer screening programs. Barcelona Euromedlab, Poster T402, 2019; May 19, Barcelona, Spain.

67. Schreuders EH, Ruco A, Rabeneck L, Schoen RE, Sung JJ, Young GP, et al. Colorectal cancer screening: a global overview of existing programmes. Gut. 2015; 64 (10): 1637-49. Review.

68. Dominitz JA, Robertson DJ, Ahnen DJ, Allison JE, Antonelli M, Boardman KD, et al. Colonoscopy vs. Fecal Immunochemical Test in Reducing Mortality From Colorectal Cancer (CONFIRM): Rationale for Study Design. Am J Gastroenterol. 2017; 112 (11): 1736-46.

69. Kaminski MF, Robertson DJ, Senore C, Rex DK. Optimizing the Quality of Colorectal Cancer Screening Worldwide. Gastroenterology. 2020; 158 (2): 404-17.

70. van Roon AH, Wilschut JA, Hol L, van Ballegooijen M, Reijerink JC, 't Mannetje $\mathrm{H}$, et al. Diagnostic yield improves with collection of 2 samples in fecal immunochemical test screening without affecting attendance.
Clin Gastroenterol Hepatol. 2011; 9 (4): 333-9.

71. Nakama H, Kamijo N, Fujimori K, Fattah AS, Zhang B. Relationship between fecal sampling times and sensitivity and specificity of immunochemical fecal occult blood tests for colorectal cancer: a comparative study. Dis Colon Rectum. 1997; 40 (7): 781-4.

72. Lee JK, Liles EG, Bent S, Levin TR, Corley DA. Accuracy of fecal immunochemical tests for colorectal cancer: systematic review and meta-analysis. Ann Int Med. 2014; 160 (3): 171-81.

73. Hernández V, Cubiella J, González-Mao MC, Iglesias F, Rivera C, COLONPREV Study Investigators, et al. Fecal immunochemical test accuracy in average-risk colorectal cancer screening. World J Gastroenterol. 2014; 20 (4): 1038-47.

74. Wong MC, Ching JY, Chan VC, Lam TY, Shum JP, Luk AK, et al. Diagnostic Accuracy of a Qualitative Fecal Immunochemical Test Varies With Location of Neoplasia But Not Number of Specimens. Clin Gastroenterol Hepatol. 2015; 13 (8): 1472-9.

75. Guittet L, Bailly L, Bouvier V, Launoy G. Indirect comparison of two quantitative immunochemical faecal occult blood tests in a population with average colorectal cancer risk. J Med Screen. 2011; 18 (2): 76-81.

76. van Roon $\mathrm{AH}$, Goede SL, van Ballegooijen $\mathrm{M}$, van Vuuren AJ, Looman CW, Biermann K, et al. Random comparison of repeated faecal immunochemical testing at different intervals for population-based colorectal cancer screening. Gut. 2013; 62 (3): 409-15.

77. Kobayashi M, Kawachi H, Pasternak S, Delgado C, Pinto $\mathrm{P}$, Ito $\mathrm{T}$, et al. Histopathologic study from a colorectal cancer screening in Chile: results from the first 2 years of an international collaboration between Chile and Japan. Eur J Cancer Prev. 2019; 28 (4): 245-53.

78. Okada T, Odagaki T, López-Köstner F, Zárate AJ, Ponce A, Kronberg U, et al. Colorectal cancer risk factors in asymptomatic Chilean population: a survey of international collaboration between Japan and Chile. Eur J Cancer Prev. 2020; 29 (2): 127-1. 